is longer in healing than with the ligature method, where the mucous membrane may be partially drawn over the top of the stump with fine silk, leaving but little raw surface.

In their technic both operations resemble each other in a great many points. The patient having been properly prepared, and anesthetized and placed in the lithotomy position, the surgeon dilates the sphincter slowly but so completely that it will not regain its tone for two or three days. A soft sponge with string attached is then passed into the rectum above the site of operation to prevent descent of any fecal matter that preceding enemas may not have brought away. Now each pile is in turn seized with a tenaculum and pulled out from the wall to make it more prominent. The mucous membrance at its base is dissected away with curved scissors. If the clamp is the method preferred, it is now applied, the blade being transverse to the axis of the gut and fitted into the dissected surface at the base, then screwed up and that portion of the tumor outside of the clamp excised. The raw surface is seared with the cautery, at dull red heat, and the clamp slowly removed.

In the ligature method a double ligature of catgut is passed through the base of the pile and each half is tied separately; the pendent portion is then removed with scissors. Care must be taken here not to cut so close to the ligature that it will afterward slip. With fine silk the mucous membrane may now be drawn over the stump as much as possible. Each pile is taken in turn, whether by clamp or ligature, after which, the sponge having been removed, the surface is dusted with some of the numerous dusting powders; modoformol seems to have given the best results in my hands. An opium suppository now being inserted, a pyramid dressing of gauze is applied, held in place by a T-bandage. After-treatment consists of opiates in addition to the suppository, when necessary, for twelve hours. Catheterization is necessary as a rule. The bowels should be kept quiet for three days and an enema of olive-oil given preceding the movement.

149 Franklin St.

\section{FIFTY-ONE MASTOID CASES. *}

FIFTY OPERATIONS, INCLUDING FOUR BRAIN ABSCESSES AND ONE PERFORATION OF THE SIGMOID SINUS.

\section{BY D. MILTON GREENE, M.D.}

Eye, Ear, Nose and Throat Surgeon to U. B. A. Hospital and Holland Home for the Aged; Lecturer to U. B. A. Training School for Nurses.

GRAND RAPIDS, MICH.

For convenience and to prevent the necessity of giving in detail the histories of a long list of cases, which would add no interest to this report, I have divided the fifty-one mastoid operations into two classes. Class one includes those which presented nothing unusual for cases of suppurative mastoiditis. Class two includes all cases not included in class one.

In class one are thirty-six operations. Of these, twenty-four followed acute suppurative otitis media. Only twelve had chronic suppurative otitis. The operations were all performed with chisel and mallet. In six cases an opening was made from the antrum into the tympanum. In only five was there swelling over the mastoid process, two of which were in children aged eighteen months and two years respectively. All had a temperature ranging from 99 to $10 €$, and all recovered. In thirty-one the diagnosis was made from

\footnotetext{
* Read before the Western Ophthalmologic Association, Feb. 10, 1899.
}

the quantity and persistency of the aural discharge, temperature, chills, pain, and especially the swelling and protrusion at the upper and posterior wall of the tympanum and auditory canal. This class, therefore, presents nothing of special interest, except the fact that a diagnosis was made in each case, operation performed, and pus found in the ceils and antra with the exception of three cases. In the cases where pus was not found, the cells and antra were in tensely congested, and I believe the operations were beneficial by their depleting effect on the parts, thereby cutting short the inflammatory process. No damage was done by the operation in any case.

In class two there are fifteen cases, each of which presents points of special interest. This includes one patient not operated on, who died, and autopsy was made confirming the diagnosis.

Case 37.-Mr. D., aged 48, gave a history of chronic aural discharge by spells for thirty years, from his left. ear; swell. ing in temporal region for several weeks, extending mostly above and anterior to the ear; temperature 102 ; pulse 100 . There was evidence of pus formation, though the swelling was tense and no fluctuation. I incised the swelling and found a hole in the skull communicating with an extradural abscess located in the middle fossa. The abscess cavity was thoroughly curretted and drained and the case recovered.

Case 38.-Miss B. had chronic aural discharge which had resisted years of treatment. She took cold from a draught of air on the side of her affected ear, had chills, pain over mastoid and in ear, and temperature ranging from 99 to 102 ; no external swelling, edema nor redness over mastoid; tympanic walls at posterior superior portion swollen. The membranes of the auditory canal at the upper and back part were swollen and inflamed. Operation, on fifth day. revealed pus in the mastoid antrum, which was freely opened and scraped. A week later a retropharyngeal abscess formed, marked by a severe chill and temperature of 105 , and when opened discharged a half ounce of pus. The patient recovered in six weeks.

Case 39.-J. S., aged 38, had pain in the left temple extending from the ear forward over the face and brow. This continued for about three weeks. He had no discharge from the ear, but his wife said he had some several years before. He improved after the first week and went to work for several days, when he was again obliged to quit work and take to his bed. At the end of three weeks from the time he was taken, he began to be delirious by spells. When asked a question he would try to answer it, but would always say something other than an answer, as for instance: "Mary" (his wife's) or some other familiar name.

A brain specialist was called, and advised the family physician and family to call me to see if he might not have some ear trouble, which had given rise to the temporal pain. When I called I found him as stated above, but also with his left eye turned outward, pupils quite large though reacting to light, temperature 98, pulse 50. I could not get a good view with the ophthalmoscope, on account of his restlessness and struggles to get out of bed, but there was choked disc to some extent; bowels constipated. He had vomited some for three days whenever he drank water. He took no food, was quite emaciated but strong. Pus was seen behind the drumhead, which was slightly inflamed in Shrapnel's membrane, and there was a little offensive pus in the auditory canal. There was no looal swelling or signs of any kind over the mastoid, and no motor paralysis excepting of the internal rectus. He had difficulty in standing, with tendency to fall to the left. $\mathrm{He}$ was anxious and excited, grasping for the bed-clothes constantly and was determined to lie on his back or left side, constantly turning his face to the right as though hunting for something on that side.

I diagnosticated brain abscess. As the brain specialist believed no abscess existed and told the family so, stating that if $I$ thought there was I could oper. 
ate, but must take all responsibilities, I opened the mastoid and found about a dram of greenish pus. I then extended the scalp incision upward and backward three inches exposing the skull an inch above and one inch back of the temporo-parietal suture. With a three-quarter-inch trephine I opened the skull an inch and a half above and an inch back of the external auditory meatus. There was no pulsation felt in the brain. With an aspirating needle on a hypodermic syringe I drew off a half-dram of pus. I then opened the membranes and incised the cerebral substance to the depth of about half an inch and evacuated two or three ounces of greenish pus, intensely offensive. The operation lasted but a few minutes, and the cavity was packed with iodoform gauze and the patient put to bed. There was considerable hemorrhage from the pia mater and capillaries of the scalp and bone; pulse was weak and rapid. The patient recovered from the anesthetic (chloroform) without vomiting. Operation was performed at 2 P.M., and at 8 P.M. he was perfectly conscious, answered questions, rose up in bed, took a tumbler of water in his hand and drank. Twentyfour hours after the operation the pulse ran up to 170 and temperature to 105 , with all the symptoms of meningitis. He died forty-four hours after operation.

Case 40.-Mr. A., aged 33, had earache nine months ago, followed by profuse purulent discharge which has continued in epite of all treatment. There were no external mastoid signs, but swelling at the posterior superior tympanic wall, and membrana tympani was half destroyed; ossicles necrotic. I removed the ossicles in April, but the discharge continued, so in July I opened the mastoid, which contained pus and softened bone. He recovered with the formation of a new drum membrane, which had been entirely removed in the first operation; has good hearing for the voice, in the operated ear, but not for a watch; had facial paralysis, from which he recovered in two months.

Case 41.-Miss J., aged 9, had earache, followed by purulent discharge from the left ear for about four years. On examination I saw a dark body in the auditory canal and tympanum, removed it and found it to be hard coal. When and how it got in the ear no one knows. The ossicles were necrotic, so I removed them. Discharge finally ceased for a time, but soon recurred. After fruitless efforts with months of treatment, I opened the mastoid, found the cells soft and full of pus, curetted, and the case made a rapid recovery. She too had facial paralysis, from which she recovered in six weeks.

Case 42.-Mr. V. V., aged 19, called at my office on account of a profuse discharge from his right ear, and especially because he was unable to open his mouth. He had no pain. There was some swelling below the tip of the mastoid. The auditory canal, near the drumbead, and the back wall of the tympanum were swollen and red. Pus was profusely pouring from the ear, so that the auditory canal would fill in ten minutes. He complained of feeling dizzy when he attempted to stand. I operated as soon as he could be prepared, and made in the soft parts, in addition to the regular section for mastoid operation, an incision obliquely upward and backward so as to get free access to the mastoid. On opening through the external plate the cells were found to be entirely destroyed by the suppurative process, and the whole mastoid process was full of pus. On attempting to scrape it out, the inner plate was found to be soft, and readily broke down over an area three-fourths of an inch by one inch, evacuating thereby an extradural abscess containing about an ounce of pus. The sigmoid sinus was in plain view for fully one inch. The tympanum was entered by chiseling away the wall between it and the mastoid, from the opening in the latter. The case made an uninterrupted recovery, and in three months he had good hearing in the ear operated upon.

Case 43.-Mr. W., aged 37, had discharge of pus from right ear for fifteen years. Temperature was normal. He had been treated much, and never cured, but had no pain. After three months' treatment, without benefit, I removed the ossicles and cleaned out all soft tissue from the tympanum. Pus continued to flow in considerable quantity and opening the mastoid was advised. After due preparation an incision was made above and behind the attachment of the auricle down to the tip of the mastoid, the lining of the auditory canal was removed as far back as the drum membrane and the ear lifted from its position. The celle and antrum were opened and found full of pus and softened bone. In removing the softened and necrotic bone the facial nerve was necessarily injured, causing facial paralysis, which yet remains. The bone was so extensively diseased that by its removal at the roof of the antrum and tympanum the dura was exposed to the extent of one-half by three-fourths of an inch. The recovery was somewhat slow, but he left the hospital in six weeks. He has been able to follow his business as foreman in a factory ever since, and has no discharge from his ear.

Case 44.--Mrs. M., aged 42, had partial deafness in both ears for years, but no earache or discharge. She had la grippe fol lowed by pain in, and discharge of pus from, the left ear, and swelling over the mastoid. I opened the mastoid antrum, and found no pus, but intense congestion. Improvement followed operation. Temperature, which was 102 to 104 before the operation, never rose above 100 , until an abscess formed in the neck, when it reached 102. The abscess was opened and she recovered promptly.

The interesting point in this case is that she recovered with perfect hearing in the operated ear, but the other (right) ear, while improved to some extent by the after-treatment, has the hearing much impaired.

Case 45.-February 21, I was called to see Miss H., 24 years of age, who had been suffering with pain in both ears for twenty-four hours. I found both drum membranes inflamed and bulging from accumulation of bloody serum in the tympanum, temperature 101 and pulse 100 . No cause could be assigned for the ear trouble. The left ear being the worst, I punctured the membrana tympani and a bloody serum flowed freely. I was again called in a few hours and opened the other drumbead with like effect. There was extreme congestion in both ears, and though the pain was relieved for the time, it soon returned in both eare. I ordered leeches and morphin to relieve the pain, and next day the temperature ranged from 98.9 to 99 . The next four days temperature was below 99 and pain continued intermittent and pus was flowing from both ears. The fifth day the temperature rose to 101, and she complained of deep-seated pain in both ears and extreme sense of pressure. Leeches had been applied. I ordered blisters over the mastoids which filled well.

On the sixth day I had counsel with several physicians, and, all symptoms being aggravated, opened the mastoid on the left side in the usual way, and found the cells and antrum full of lymph and blood and some small deposits of pus. I opened from the antrum into the middle ear through the bony wall, then made a Wilde's incision on the right side. Pain was relieved on the operated side, but continued on the right.

At the operation we noticed, just in front of the tragus, a red spot as large as a 25-cent piece, surrounding a recent leech-bite, but thought it nothing serious. At night, to my surprise, it had spread to the size of three inches in diameter, extending over the cheek and eye, and we had a case of erysipelas which spread over the face, head, neck, and in a few days had extended down the back as far as the last dorsal vertebra, but had not attacked the wounds over the mastoids, which were made within a portion of skin which had been blistered by cantharides. As the patient was delirious and seemed to be growing worse, and the erysipelatous process was steadily going on, not having been checked by carbolic acid, subcuta. neous injections of boric acid, etc., I circumscribed it by cantharideal collodion. This was suggested by the fact that though the whole head was enveloped by erysipelas, the mastoids which had been blistered escaped. This treatment cut short the spread of erysipelas, and the inflammation never went beyond the blister, which extended in a line over the breast and back, encircling the whole body. In a few days the erysipelas was entirely cured. At the end of thirty days she still had severe pain in her neck and arm and some in the right ear; pulse 100 and temperature 101.4.

The symptoms caused by erysipelas had made it impossible for me to judge what part of her fever, 
delirium and pain was due to the ear and mastoid, and what part to the erysipelas; but as her temperature was above 101 and erysipelas cured, with pus flowing from her right ear, with swelling at the upper back part of the typanum bulging forward and downward, I opened the mastoid and evacuated two or three drams of pus, and the pain res relieved. From this time she went on to complete recovery. I removed a small sequestrum of necrotic bone from the wound in the left mastoid during this time. She was completely deaf in both ears before the operation, we being obliged to write what we said to her, but the ear first operated was soon restored to hearing, while the hearing in the other ear is still defective. She had facial paralysis, from which she recovered.

If I had such a case again I should open the mastoid on both sides at the time of the first operation.

Case 46.-Baby N., aged 3 years, had extreme swelling in the region of the right ear, which existed for a week, when the family physician called me to see it. 1 made a free incisior and evacuated a large quantity of pus, and ordered that it be kept drained and cleaned by irrigation.

I was surprised, a year later, to learn from the father that the wound was still open and discharging pus, but otherwise the child was perfectly well. I advised an examination, but before they got around to see me again a piece of necrotic bone of considerable size was expelled and the wound healed rapidly.

Case 47.-Baby B., aged 18 months, was taken with fever and was ill for a wook, when the father, who was a physician, noticed swelling in the neck and mastoid region. There was and had been no discharge from the ear. I incised the mastoid coverings freely and evacuated four or five drams of pus, and the case recovered rapidly, without discharge from the ear ever taking place.

Case 48.- Mr. T., age 40, had suffered from chills and fever for three weeks when he came under my observation on account of a profuse and offensive discharge from his left ear. There had been suppurative otitis in the left ear for twelve years. The pulse was 60 , temperature 99 , pupils normal and no choked disc. $\mathrm{He}$ was intensely nervous and restless, and had not slept for several days. The mastoid was opened and pus found in the celle and antrum. The roof of the tympanum was soft, and was scraped away, leaving the dura over that portion of the temporal lobe exposed. As there was no pulsation to be felt in the brain I paseed an aspirating needle in and found an abscess in the temporal lobe, containing an ounce of pus. The cavity was reached by opening the dura to and the abscess seemed to be walled off. A drainage tube was inserted after thorough irrigation, and the case went on to recovery.

Case 49.-Mrs. W., age 42, had acute otitis media with fre. quent chills. The pulse ranged from 90 to 120 . She had not much pain after the first few days, but at the end of about seven days had a severe chill and temperature of 105 . Soon after this she had metastatic abscesses form in her legs; they continued to form and she had frequent severe chills followed by high temperature, which would again go down to 99 and remain so until after another chill. An early diagnosis of mastoid suppuration was made and an operation refused.

Later a diagnosis of perforation of the sigmoid sinus was made, but no operation was allowed. She died at the end of seven weeks. Autopsy confirmed the diagnosis, the antrum and cells being full of pus, and the sigmoid sinus filled with pus and clot.

Case 50.-M. W., aged 6, had scarlet fever, and, toward the end of the disease, severe suppurative otitis media supervened and she became entirely deaf. The discharge continued profusely and she had chills with a temperature ranging as high as 105 .

I was called and diagnosed mastoid complication and advised operation. After a week's delay, and when she was growing weaker, then being but a mere skeleton, the family wanted an operation. Both ears were equally affected. I opened both mastoids at the same operation and evacuated a quantity of pus. The temperature went down to 100 the next day and only once did it reach 102, when the drainage had become imperfect. She made a good recovery after several weeks. As she was totally deaf before the operation as a result of the scarlet fever and extension of the inflammation to the labyrinth, she is still deaf.

Case 51.-L. V., aged 26, was taken with pain in his left ear, and had swelling around the ear and temple extending finally to his neck, jaw and face. Later a retropharyngeal abscess developed and the swelling on the outside extended along the large vessels down to the clavicle. The abscess in the throat broke and discharged pus. At the end of three weeks, an abscess developed on the left side at the angle of the eighth rib, which was incised and discharged several ounces of pus. At the end of five weeks 1 was called to see the case, and found an abscess over the heart which encroached upon the left chest. Pus was distinctly seen behind the drum membrane.

Three days before I saw him he had a severe chill and since the day after the chill had been unable to utter but one word, which he repeated whenever asked a question or if he wished to call attention; that word was "no." The pupils were large, but the right larger than the left. They reacted promptly to light. There was no choked disc, and no paralysis. I diagnosticated involvement of the mastoid, medias. tinal abscess and brain abscess at the angle of the fissure of Sylvius, above the fork. The next day the mastoid and tympanum were opened and pus evacuated. The abscess over the heart was incised; it con. tained fully a quart of pus. The patiant suffered no shock from the operation and improved for a few days. I insisted upon opening the skull, but was unable to get the consent of the family until the patient became paralyzed in the face, arm and leg of the right side; then, five weeks after the first operation, I opened the skull and verified the diagnosis by evacuating three ounces of pus from a brain abscess. ${ }^{1}$

As for the particular form of operation adopted in the cases herein reported, I would say that no hard and fast rule can be laid down for mastoid operations. I usually perforate the cells and antrum with a large-sized hollow chisel, in the depression one-quarter to one-half inch back of the auditory canal. If pus is found, and it is deemed necessary, I proceed from this opening to remove as much of the bone as necessary, always bearing in mind the important anatomic structures. It would be useless for me to enter into the technic of mastoid operations, for that is thoroughly covered in other papers and in text-books.

\section{METHODS OF PREVENTION IN SMALLPOX.*}

\section{BY SOLON R. TOWNE, A.M., M.D.}

$$
\text { OMAHA, NEB. }
$$

In the face of the conditions existing in various parts of the country this past winter this theme was chosen for a clear statement of methods and to form a subject for discussion. No new principle is to be presented. We have only to trust to four well-estab. lished agencies, namely, diagnosis, quarantine, vaccination and disinfection.

An early and positive diagnosis in an individual case of variola is not always possible. Anders, in his last edition, declares that "This difficulty is sufficient occasionally to test the skill of the most astute diagnostician, and the sooner this fact is understood and

1 A full report of this case will be given in a paper before the Section on Surgery and Ophthalmology, American Medical Association, at Columbus in June.

Mo., March 16, 1899. 